\title{
Research on equipment maintenance data recording method based on E-R diagram model
}

\author{
Jin-wei Qiao ${ }^{\mathrm{a},},{ }^{*}$, Xiao-hu Yin ${ }^{\mathrm{b}}$, Gongmin Tang ${ }^{\mathrm{c}}$ \\ Unit 72465 of People's Liberation Army, Jinan, China, 250022

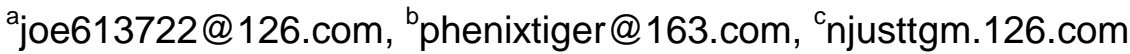

Keywords: E-R graph relation, Equipment Maintenance, Data Recording, Data Mining.

Abstract. For existing equipment maintenance records, normative is not so strong. Worse more, the text-based description always makes post data mining impossible or difficult. In order to realize the equipment maintenance information, this paper imports the basic idea of E-R (Entity-Relation) graph model into numbering separate parts and assembly relations. The Roche mechanical turbocharger was taken as an example to describe how to operate the numbering and display the powerful potential in the post data-mining process.

\section{Introduction}

All equipment exists in the form of assemblies. Common method that describes assembly information includes combination directed graph[1], hinder relation graph[2], hierarchy relation graph[3], constraint model[4], relationship model, joint relation graph, multi tuple and so on. The application of methods mentioned above was aimed to improve to save assembling steps, plan assembling methods and raise assembling efficiency in engineering practice. Among them, the most classical and representative description method is the so called combination directed graph.

It saves the process information of model building in directed edges, so that more information can be provided to cut set analysis for assembly sequence planning.

With the development of computer technology and information technology, the amount of information presented an exponential growth, some useful information or knowledge could be extracted, and thus decision-making was improved.

Traditional analytical methods have long gone beyond the real demands. A combination of statistical, database, machine learning and the like techniques, data mining techniques can be fully applied to the field of equipment maintenance and support. It will provide strong support to maintain the support system and decision-making in information battlefield environment. Basis of data mining is to have a large number of standardized, systematic data. The input data comes from a large number of front-line staffs who give real-time input in their repair process. However, the input data from equipment maintenance requires meeting certain rules. Nevertheless, data recording always follows traditional ways that only needs to fill some failure modes in tables shown in Tab. 1.

Without strict grammar rule or constraint requirement, all data were input by hand, heavily depending on personal experience or habit. The text description information filled in blanks in Fig.1 is obvious and intuitive for human, but for computer it is extremely hard to understand or to process. Mass of such text information, as a result piles up to be a big trouble for post massive data mining. 
Table 1 Equipment failure record table

\begin{tabular}{ccc}
\hline NO. & 1 & 2 \\
\hline Date & 07.03 .2015 & 09.20 .2015 \\
\hline Failure time & Roche mechanical turbocharger & Roche mechanical turbocharger \\
\hline Failure location & Nut old and loosen & Spring fracture \\
\hline Failure cause & Change nut & Change spring \\
\hline Treatment & 07.03 .2015 & 09.22 .2015 \\
\hline Recorder & James & Ethan \\
\hline Repair date & 07.03 .2015 & 09.22 .2015 \\
\hline
\end{tabular}

Combing the E-R graph model structure and assemble characteristic, this paper propose a standard equipment data recording mode. A practical Roche turbo charger was cited as an example to classify the recording mode. The result of this paper built up a concrete basis for post data mining.

\section{E-R graph model}

E-R graph model is composed of entity, characteristic and relation. Among them, the entity means a data user that in software system can be a physical item in real life such as people, animal, object, table, department, project, and so on. A same kind of entities makes up an entity set. The meaning of entity can be represented by entity type that defines entities in an entity set. All characteristic of entities is named as attribute which can be name, gender, address, phone number and the like. In an entity, the so called 'entity identifier' is the only one that can represent the entity attribute and attribute set. One entity has one entity identifier, also called 'primary key'. In E-R graph, entity attribute is circled by an ellipse. Meanwhile, the one has underline is what we called identifier. Not an entity could exist independently in our real world. There are always kinds of relations among entities. For example, someone works in a department, among which entity includes 'someone' and 'department'. Many different connections exist among them.

Equipment maintenance informatization requires accumulating lots of original data which also needs saving, arranging, checking and post processing. The development of data base technology offers quality technique support. For now, the entity-relation, i.e. E-R model has become the most successful one in data base technology. The model can represent entities and relations. It is an abstraction of real world, has nothing with computer system, and an understandable data description way for customers.

\section{Practical application}

Part numbering In E-R graph model, each part that needs to be repaired is an independently existing entity. On the other hand, each part has and only has one graph numbering. Therefore, the rule for numbering engineering graphs can be referred. However, some change has to be made in the aim of adapting equipment maintenance process better. The changes mainly include:

a. Each part (includes standard ones) has its own unique number. Same parts have totally different number due to their different assembly location.

b. Treat parts and their assembly relation equally, give uniform number.

The Roche mechanical turbo charger (RMTC) is cited as an example here. Fig. 1 shows its explosion graph. 


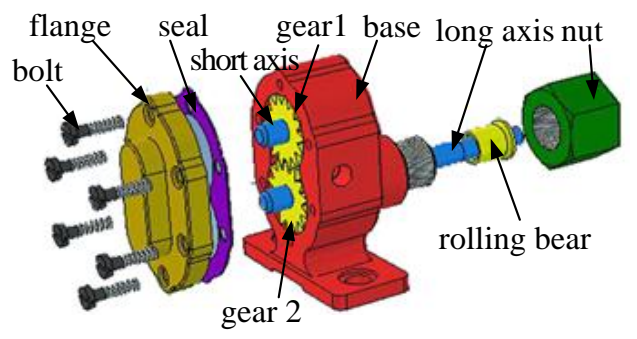

Figure 1 Explosion graph of RMTC

Now number all parts of RMTC. So do vehicle, engine, turbo charger and assembly. For example, the flange shown in Fig. 1 can be numbered as A1013E121T027P001. Here, 1013 is the last four number of vehicle chassis number, 121 is the engine number, 027 is the turbo charger number, and 001 is the part number in its own assembly. Other numbering for left parts in turbo charger is list in Tab. 2.

Table 2 Part numbering in RMTC

\begin{tabular}{ccc}
\hline Part name & Part number & Failure number \\
\hline flange & A1013E121T027P001 & L001 \\
seal & A1013E121T027P002 & L002 \\
short axis & A1013E121T027P003 & L003 \\
gear 1 & A1013E121T027P004 & L004 \\
base & A1013E121T027P005 & L005 \\
long axis & A1013E121T027P006 & L006 \\
nut & A1013E121T027P007 & L007 \\
sliding bear & A1013E121T027P008 & L008 \\
gear 2 & A1013E121T027P009 & L009 \\
bolt 1 & A1013E121T027P010 & L010 \\
bolt 2 & A1013E121T027P011 & L011 \\
bolt 3 & A1013E121T027P012 & L012 \\
bolt 4 & A1013E121T027P013 & L013 \\
bolt 5 & A1013E121T027P014 & L014 \\
bolt 6 & A1013E121T027P015 & L015 \\
\hline
\end{tabular}

It is worth noticing that six same bolts were separately given different numbers in Tab. 2 for their different assembly locations. So were two same gears. The benefit is to quicken the failure location during equipment maintenance.

Connection type numbering When the assembly fails, it may be some part problem, or there may be destroyed assembled relationships. Therefore, assembly relationships need to be numbered on the basis of fully numbered parts. Take equal consideration of entities and their relationships, as well as treat them equally. The idea both comes from E-R relation model. 
Existing assembly relationships could be classified roughly into 14 types, list in Tab. 3 .

Table 3 Connection type numbering table

\begin{tabular}{|c|c|c|c|}
\hline Connection type & Number & Connection type & Number \\
\hline Coincide & R01 & Angled & R08 \\
\hline Parallel & R02 & Cam & R09 \\
\hline Vertical & R03 & Hinge & R10 \\
\hline Tangent & R04 & Gear & R11 \\
\hline Coaxial & R05 & Rack and pinion & R12 \\
\hline Solid & R06 & Spiral & R13 \\
\hline Distanced & R07 & Universal joint & R14 \\
\hline
\end{tabular}

Assembly relation numbering The assembly relationship between every two parts in assemblies corresponds to entity relationship in E-R model. Each assembly relationship connects two parts in certain, while each part for sure has at least one assembly relationship attached. That is to say every part as an entity has a one-to-many relationship with any other parts.

As shown in Fig. 2, the base that was set as the most basic part in RMTC assembly was fixedly connected with outside. Based on this, sliding bear, short axis, seal and long axis all have assembly connection with base, in the form of coincide and coaxial. Although the connection type between nut and base was coincided face to face, the coaxial connection was realized by clearance fit with sliding bear. Six bolts installed in outer flange in clockwise direction were connected with assembly in the form of coincide and coaxial type.

No entity attribute is referred in this paper, nor does the building of relationship depend on entity attribute. All directly rise from entity itself, which is because assembly relationships in mature product are quite stable with little chance of frequent change. Therefore, assembly relation among parts is so obvious that building relations doesn't rely on complicated attribute. It is more convenient to set relationship directly based on entities.

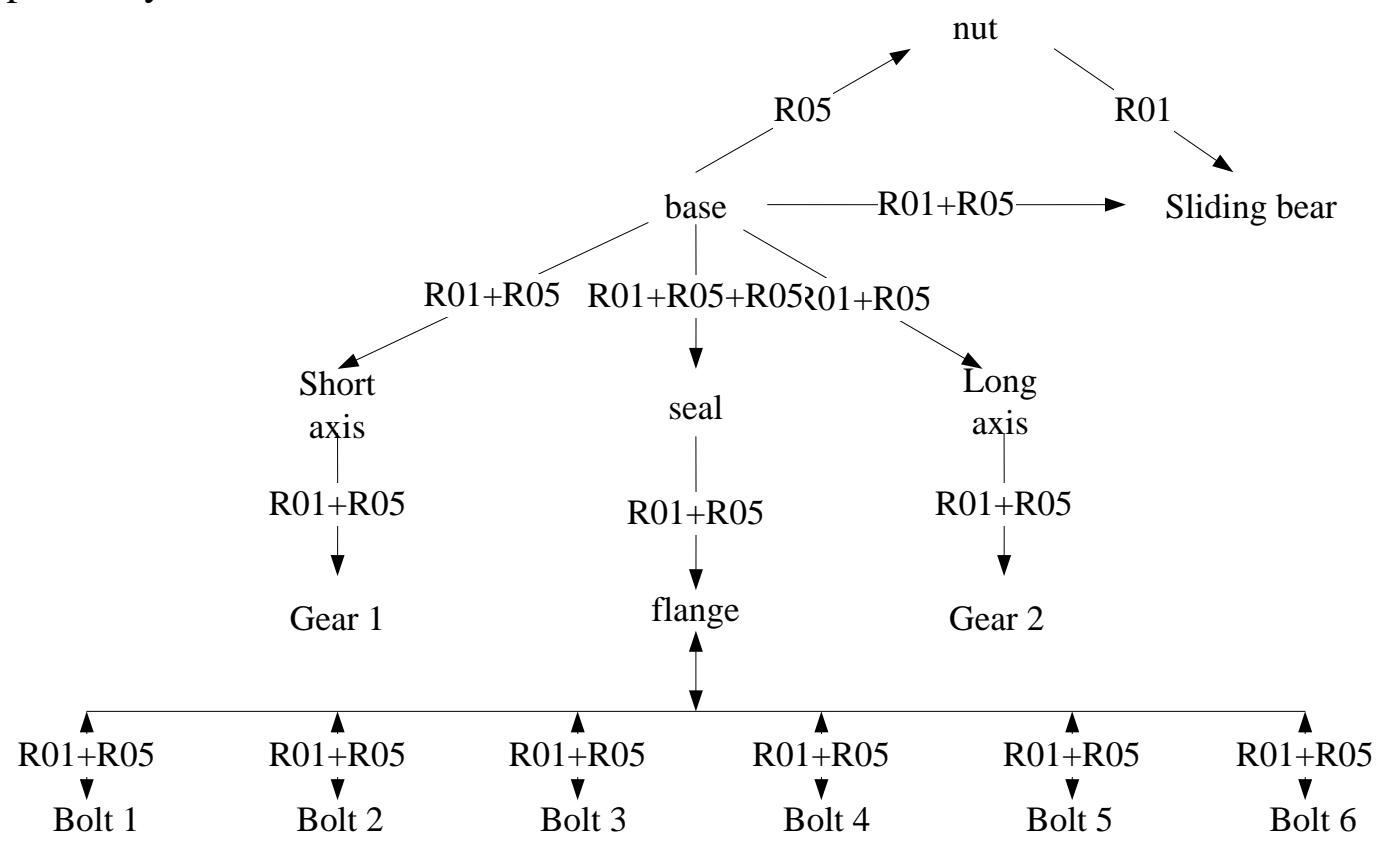

Figure 2 RMTC assembly relationship graph

Fig. 2 describes the assembly relationship among parts in RMTC. Based on this, Tab. 4 lists all numbers that define every relationship. 
Table 4 RMTC assembly relationship number

\begin{tabular}{cccc}
\hline Part 1 & Part 2 & Assembly type & Number \\
\hline P005 & outside & R06 & L016 \\
P007 & P005 & R05 & L017 \\
P003 & P005 & R01 & L018 \\
P003 & P005 & R05 & L019 \\
P002 & P005 & R01 & L020 \\
P002 & P005 & R05 & L021 \\
P006 & P005 & R01 & L022 \\
P006 & P005 & R05 & L023 \\
P004 & P003 & R01 & L024 \\
P004 & P003 & R05 & L025 \\
P001 & P002 & R01 & L026 \\
P001 & P002 & R05 & L027 \\
P006 & P009 & R01 & L028 \\
P006 & P009 & R05 & L029 \\
P001 & P010 & R01 & L030 \\
P001 & P010 & R05 & L031 \\
P001 & P011 & R01 & L017 \\
P001 & P011 & R05 & L018 \\
P001 & P012 & R01 & L019 \\
P001 & P012 & R05 & L020 \\
P001 & P013 & R01 & L021 \\
P001 & P013 & R05 & L022 \\
P001 & P014 & R01 & L023 \\
P001 & P014 & R05 & L024 \\
P001 & P015 & R01 & L025 \\
P001 & P015 & R05 & L026 \\
\hline
\end{tabular}

So far, the numbering process has been finished for parts and their relations. What follows is a detailed case, and database technique was applied to discuss the case.

Case study The case is that the third bolt was found loosen during one maintenance process. It means the coinciding set, i.e. L019 was destroyed between bolt 3 and flange. If the third bolt itself broke, the damage number is L012. Following this way can fill up the equipment maintenance statistic form shown in Tab. 5. 
Table 4 equipment maintenance statistic form

\begin{tabular}{ccccc}
\hline Serial Number & 1 & 2 & 3 & 4 \\
\hline Date & 08.07 & 09.0 & 09.1 & 09.07 \\
& & 7 & 7 & \\
Failure date & 07.03 & 07.0 & 08.0 & 07.03 \\
Failure location & T027 & T027 & T027 & T027 \\
Failure cause & L019 & L012 & L019 & L019 \\
Repair date & 08.07 & 09.0 & 09.2 & 09.08 \\
Recorder & James & Tom & Joe & Lemon \\
\hline
\end{tabular}

All failure messages can be put into database one by one in the way shown in Tab. 5. It makes post data analysis and data mining possible.

In the post data analysis, many scientific conclusions can be got such as which part has the most frequent failure, which assembly relationship has the most breaking chance, how much time is required for each particular type of failure, which season always has most failure, which technician has the lowest repair rate. All these results could be used to help make maintenance decisions.

\section{Conclusions}

1. In order to digitalize the equipment maintenance, this paper proposes a method to describe assembly in E-R relationship language based on E-R graph model theory.

2. Select the RMTC as a research object, number each part and each assembly relation, and then describe failure message in number code.

The advantage on post data processing was demonstrated by a case study.

4. The method proposed in this paper also fit circuit system. Parts mentioned here can be replaced by electric unit, and assembly relationship corresponds to linkages among different electric unit.

5. The result of this paper can also give a clear guide about how to match maintenance tools. More tools need to match parts or assembly relationship with most frequent failure rate.

\section{References}

[1] Yili Fu, Lizhong Tian, Zhengwei Dong, et. Research on expression method for directed graph in assembly relationship [J]. Computer Integrated Manufacture System -CIMS, 2003(02): 149-153. [2] H W R, Claude L J. Geometric Reasoning about Mechanical Assembly [J]. Artificial Intelligence, 1994, 71: 371-396.

[3] Heedong K, Kunwoo L. Automatic Assembling Procedure Generation from Mating Conditions[J]. Computer Aided Design, 1987, 19(1): 3-10.

[4] Tong X, Liu J, Guo R, et al. Three-dimensional magneto telluric regularized inversion based on smoothness-constrained model[J]. Transactions of Nonferrous Metals Society of China, 2014, 24:

509-513. 